\title{
Time Scales in Spectator Fragmentation *
}

C. Schwarz ${ }^{\dagger}{ }^{a}$, S. Fritz $^{a}$, R. Bassini ${ }^{b}$, M. Begemann-Blaich ${ }^{\ddagger}$, S.J. Gaff-Ejakov ${ }^{c}$, D. Gourio ${ }^{a}$, C. Groß $\aleph^{a}$, G. Imméd ${ }^{d}$ I. Iori ${ }^{b}$, U. Kleinevoß ${ }^{a} \S$, G.J. Kunde ${ }^{c}$ ฯ , W.D. Kunze ${ }^{a}$, U. Lynen $^{a}$, V. Maddalena ${ }^{d}$, M. Mahi ${ }^{a}$, T. Möhlenkamp ${ }^{e}$, A. Moroni ${ }^{b}$, W.F.J. Müller ${ }^{a}$, C. Nociforo ${ }^{d}$, B. Ocker ${ }^{f}$, T. Odeh ${ }^{a}$, F. Petruzzelli ${ }^{b}$, J. Pochodzalla ${ }^{a}{ }^{* *}$, G. Raciti $^{d}$, G. Riccobene ${ }^{d}$, F.P. Romano ${ }^{d}$, A. Saija ${ }^{d}$, M. Schnittker ${ }^{a}$, A. Schüttauf ${ }^{f \dagger}{ }^{\dagger}$, W. Seidel ${ }^{e}$,

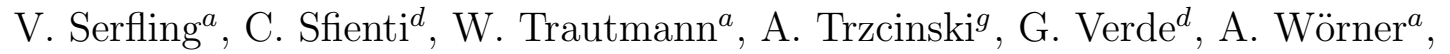
Hongfei $\mathrm{Xi}^{a}$, and B. Zwieglinski ${ }^{g}$

${ }^{a}$ Gesellschaft für Schwerionenforschung, D-64291 Darmstadt, Germany

${ }^{b}$ Istituto di Scienze Fisiche, Università degli Studi di Milano and I.N.F.N., I-20133 Milano, Italy

${ }^{c}$ Department of Physics and Astronomy and National Superconducting Cyclotron Laboratory, Michigan State University, East Lansing, MI 48824, USA

${ }^{d}$ Dipartimento di Fisica dell' Università and I.N.F.N., I-95129 Catania, Italy

${ }^{e}$ Forschungszentrum Rossendorf, D-01314 Dresden, Germany

${ }^{f}$ Institut für Kernphysik, Universität Frankfurt, D-60486 Frankfurt, Germany

${ }^{g}$ Soltan Institute for Nuclear Studies, 00-681 Warsaw, Hoza 69, Poland

Proton-proton correlations and correlations of $\mathrm{p}-\alpha, \mathrm{d}-\alpha$, and $\mathrm{t}-\alpha$ from spectator decays following ${ }^{197} \mathrm{Au}+{ }^{197} \mathrm{Au}$ collisions at $1000 \mathrm{AMeV}$ have been measured with an highly efficient detector hodoscope. The constructed correlation functions indicate a moderate expansion and low breakup densities similar to assumptions made in statistical multifragmentation models. In agreement with a volume breakup rather short time scales were deduced employing directional cuts in proton-proton correlations.

PACS numbers: 25.70.Pq, 21.65.+f, 25.70.Mn

\section{INTRODUCTION}

Understanding the simultaneous formation of many fragments helps to gain an insight into multifragmentation. Densities lower than ground state density of nuclei are a prerequisite of statistical models describing multifragmentation [1,2]. While static statistical models form fragments in an expanded volume breakup, the dynamic statistical model

\footnotetext{
*Work supported by the European Community under contract ERBFMGECT-950083

$\dagger$ Work supported by the Deutsche Forschungsgemeinschaft under contract Schw510/2-1

${ }^{\ddagger}$ Work supported by the Deutsche Forschungsgemeinschaft under contract Be1634/1-1

$\S$ Present address: Fachbereich Physik, Bergische Universität, D-42119 Wuppertal, Germany

\Present address: Dept. of Physics, Yale University, New Haven CT 06512, USA

"Work supported by the Deutsche Forschungsgemeinschaft under contract Po256/2-1

**Present address: Max-Planck-Institut für Kernphysik, D-69117 Heidelberg, Germany

${ }^{\dagger \dagger}$ Present address: Max-Planck-Institut für Physik, D-80805 München, Germany
} 


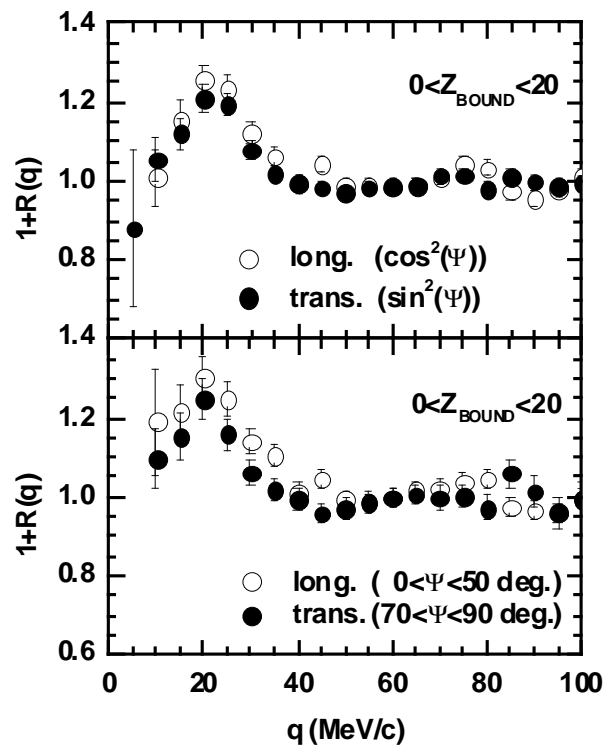

Figure 1. Proton-Proton correlation-functions for central collisions. The top panel shows directional selections generated with harmonic weights, the bottom panel shows directional selection of pairs of protons, whose angular ranges of the angle between relative and sum momentum are indicated in the figure.

[3, 4] combines an expansion with surface emission until the volume breakup of the remaining source occurs. Interferometry-type methods are widely considered as a valuable tool in determining the space-time extent of such a source and recently, spectator remnants of the reaction $\mathrm{Au}+\mathrm{Au}$ at $1 \mathrm{AGeV}$ incident energy were found to break up at densities, considerable lower than ground state density [5]. In that analysis a instantaneous volume breakup was assumed. In such a breakup the mean time difference between the emission of two fragments is assumed to be short, due to fluctuations of the breakup time.

In this article we report on results of correlation measurements of protons from the target spectator following the collisions of $\mathrm{Au}+\mathrm{Au}$ at $1 \mathrm{AGeV}$ incident energy. Coincidences between protons were used to construct correlation functions. The results are found to be consistent with low breakup densities with values close to those assumed in the statistical multifragmentation models and short emission time differences with values close to those anticipated for volume breakup.

\section{EXPERIMENT}

Targets, consisting of $25 \mathrm{mg} / \mathrm{cm}^{2}$ of ${ }^{197} \mathrm{Au}$ were irradiated by an $1 \mathrm{AGeV} \mathrm{Au}$ beam delivered by the Schwerionen-Synchrotron (SIS) at GSI in Darmstadt. For the results, presented here, we employed one multi-detector hodoscope, consisting of a total of $96 \mathrm{Si}$ $\mathrm{CsI}(\mathrm{Tl})$ telescopes in closely packed geometry. The hodoscope covered an angular range $\Theta_{\text {lab }}$ from $122^{0}$ to $156^{0}$ with the aim of selectively detecting the products of the targetspectator decay. Each telescope consisted of a $300 \mu \mathrm{m}$ Si detector with $30 \mathrm{x} 30 \mathrm{~mm}^{2}$ active area, followed by a $6 \mathrm{~cm}$ long $\mathrm{CsI}(\mathrm{Tl})$ scintillator with photodiode readout. The distance to the target was $60 \mathrm{~cm}$. The products of the projectile decay were measured with the time-of-flight wall of the ALADIN spectrometer [6] and the quantity $Z_{B O U N D}$ was determined event-by-event. $Z_{B O U N D}$ is defined as the sum of the atomic numbers $Z_{i}$ of all fragments with $Z_{i} \geq 2$. It reflects the variation of the charge of the primary spectator system and serves as a measure for the impact parameter. 


\section{DATA ANALYSIS}

\subsection{Correlation functions}

The correlation functions were constructed dividing the spectrum of relative momenta of two coincident particles by the spectrum of pairs from different events. One observes at a relative momentum of $q \approx 20 \mathrm{MeV} / \mathrm{c}$ a maximum of the correlation function, which is dominated by the attractive singlet scattering between two protons. The height of the maximum is inversely related to the relative distance between two protons and hence, the diameter of the source for simultaneous emission [7,8]. Different emission times of the protons cause an elongation of the source in the direction of the hodoscope [9]. Since two protons in the perpendicular direction are closer, they suffer due to the Pauli blocking a stronger suppression. We selected pairs of protons with directional cuts on the angle $\Psi$ between the sum momentum $\mathbf{P}$ and the relative momentum $\mathbf{q}$. The choice of the sum momentum as reference direction is motivated by the tilted source elongation with respect to the beam axis. The notion for longitudinal $(\mathbf{P} \| \mathbf{q})$ and transversal $(\mathbf{P} \perp \mathbf{q})$ correlation functions, therefore, differs from what is commonly used in high energy physics. Instead of using cuts on the angle $\Psi$ between sum momentum and relative momentum we employed for the first time a harmonic weight for each event. We used $\cos ^{2}(\Psi)$ and $\sin ^{2}(\Psi)$ as weight for the longitudinal and transversal correlation function, respectively.

The proton-proton correlation-functions are shown in Fig. 11 for the most central impact parameter bin. We used protons with energies $E>20 \mathrm{Mev}$ in order to prevent distortions from sequential decay [10]. The correlation functions show a pronounced peak due to the final state interaction at $q=20 \mathrm{Mev} / \mathrm{c}$ relative momentum. The longitudinal selection exhibits at lower relative momenta, $q<50 \mathrm{MeV} / \mathrm{c}$, correlations which are $\approx 5 \%$ stronger compared with the transversal selection. For a source with Radius $R=7 \mathrm{fm}$ one can roughly estimate from the uncertainty relation the relative momenta where antisymmetrization effects should take place as $q=\sqrt{\mathbf{q}^{2}}<\sqrt{3} * \hbar / r \approx 50 \mathrm{MeV} / \mathrm{c}$. The bottom panel of Fig. 1 serves as comparison between the two methods for generating directional cuts. Here, pairs of protons were selected according to the orientation between sum momentum and relative momentum as it was done in analyses performed before [9]. Employing harmonic weights gives the advantage to use the full data set for one directional

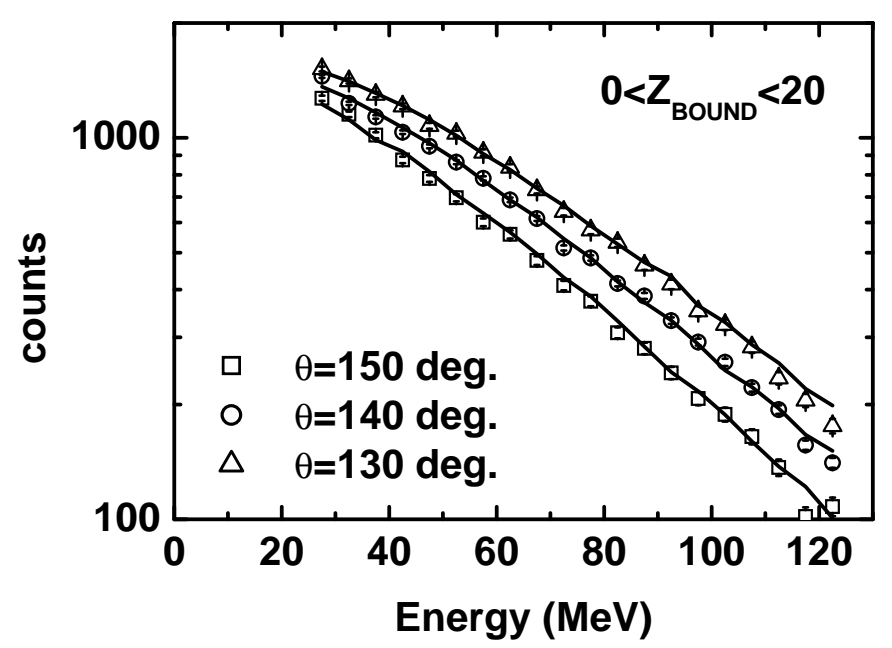

Figure 2. Energy spectra of protons at angles of 130, 140, and $150 \mathrm{deg}$. in the laboratory system. 


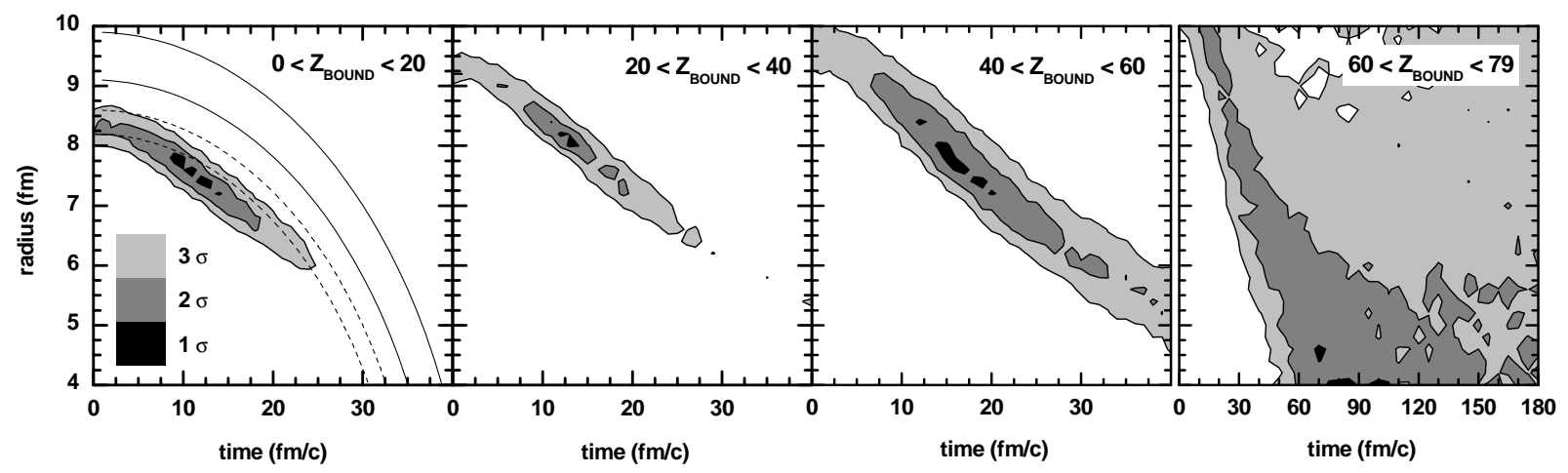

Figure 3. $\chi^{2}$ distributions as a function of radius and emission time of the emitting source for four impact parameter ranges indicated by the $Z_{B O U N D}$ ranges in the panels. The lines in the left panel are explained in the text.

selection, while for the hard cuts less than half of the events are used. In detector setups with limited angular acceptance a hard transversal cut limits the statistic for large relative momenta and makes the normalization more difficult. This eg. can be seen from the larger error bars of the transversal correlation function in the bottom panel of Fig. 1 for relative momenta $q>60 \mathrm{MeV} / \mathrm{c}$. Here, many of the proton pairs with large transversal relative momenta miss the hodoscope.

\subsection{Monte-Carlo simulations}

The analysis of the p-p correlation functions was performed with the Koonin-Pratt formalism [7, 8]. Particles were chosen to be emitted randomly from the volume of an uniform sphere and their velocities were sampled according to a Maxwell-Boltzmann distribution. An additional velocity component was added, which simulates the Coulomb repulsion dependent on the location of the particle within the source. The strength of this component was chosen recursively, in order to agree with the later extracted source radii. From the measured proton-energy spectra (Fig. 2) one can observe that the emission probability of the source is non isotropic. We modeled this anisotropy by assuming

\begin{tabular}{cccccc}
\hline$Z_{\text {BOUND }}$ & $\begin{array}{c}T \\
(\mathrm{MeV})\end{array}$ & $\begin{array}{c}P_{\text {bounce }} \\
(\mathrm{MeV} / \mathrm{c})\end{array}$ & $\begin{array}{c}P_{\text {Coulomb }} \\
(\mathrm{MeV} / \mathrm{c})\end{array}$ & $\begin{array}{c}\mathrm{v}_{\text {stop }} \\
(\mathrm{c})\end{array}$ & Mult. \\
\hline $0-20$ & 26 & 105 & 80 & 0.020 & 6.1 \\
$20-40$ & 23 & 85 & 120 & 0.015 & 6.2 \\
$40-60$ & 19 & 80 & 140 & 0.010 & 5.8 \\
$60-79$ & 16 & 80 & 160 & 0.005 & 4.2 \\
\hline
\end{tabular}

Table 1

Source parameters for the MC-calculation of the protons: given are the temperature $T$, the Gaussian momentum width of the transversal movement of the source, the maximum momentum due to the Coulomb repulsion, the Gaussian width of the proton-multiplicity spectrum, and the velocity of the source in beam direction $\mathrm{v}_{\text {stop }}$. 
a sideward movement (bounce) of the source perpendicular to the beam, which causes a reaction plane. It influences slightly the correlation functions by the event mixing technique which we used: the choice of a pair of protons from events with different reaction planes causes an additional small relative momentum between the momenta. Therefore, the relative momentum spectrum of the mixed events is slightly shifted to larger values compared to the spectrum of the coincident proton pairs. The correlation function then shows a slightly decreasing slope. Since our experiment lacks an azimuthal symmetric solid angle coverage, we were not able to determine the reaction plane and to rotate the different events in the same plane to avoid these effects 11. We rather preferred to introduce the reaction plane in the Monte-Carlo calculations. In order to simulate the hit probability of the hodoscope for different bounce values, we adjusted the multiplicity of the event such that it resembles the measured proton-multiplicity spectrum after applying the experimental filter. The extracted source parameters are given in Tab. 1.

\section{RESULTS}

We varied the radius of an uniform density distribution and the Gaussian emission time of the protons. The time distribution is guided by the idea of a statistically independent emission of different emitters within the source, rather than the decay of an excited state (see eg. [12]). The simulated correlation functions were used to perform a $\chi^{2}$-test algorithm in the relative momentum region of $10 \leq q \leq 35 \mathrm{MeV} / \mathrm{c}$. The results are presented in Fig. 3. The grey scale of the shading denotes within which standard deviation the space-time point is. The minima in Fig. 3 show approximately constant source radii and surprisingly short emission times. For the most peripheral bin a radius and emission time could due to the low statistics of the correlation function not be deduced. The experimental correlation functions (symbols) and the simulations (lines) for the best $\chi^{2}$ are shown in Fig. 4 , left panel. They agree well with their simulations. The

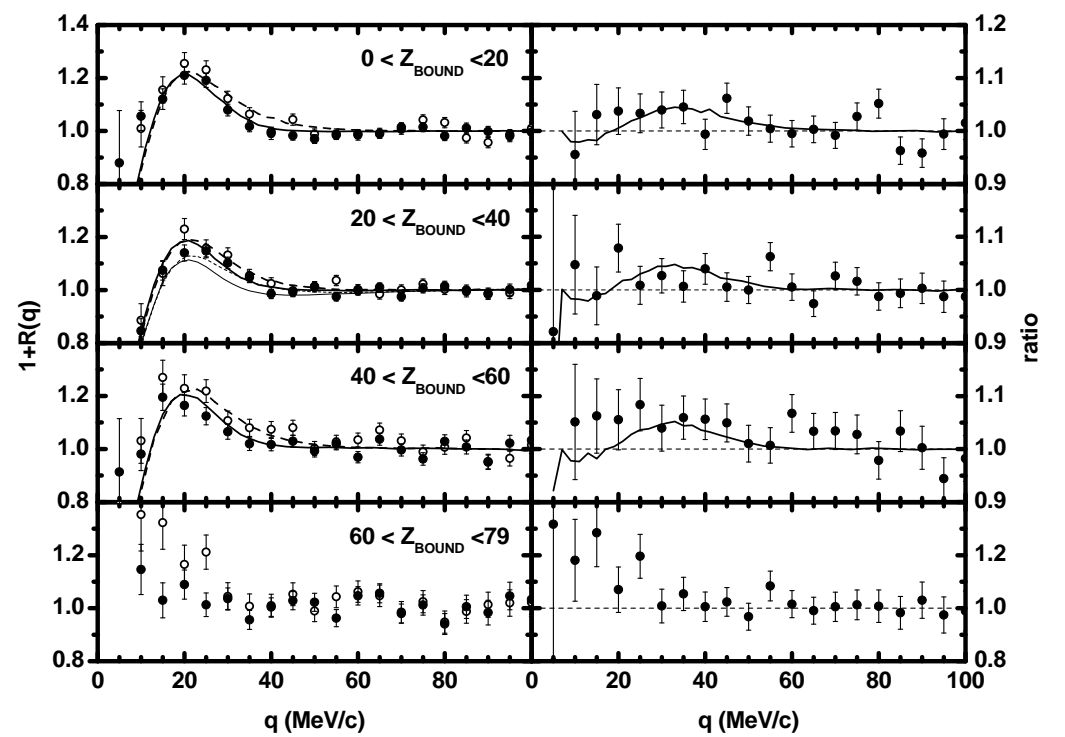

Figure 4. The left panel shows experimental longitudinal (open symbols) and transversal (closed symbols) correlation functions and results of MC-simulations (lines). The right panel compares the their experimental ratios (symbols) with the results of the MC-simulation (lines). 
minimum $\chi^{2}$ values per degree of freedom are within the range of $1.3 \leq \chi^{2} /$ d.o.f. $\leq 2.4$. The right panel in Fig. 1 presents the ratios between longitudinal and transversal correlation functions. One recognizes the weak enhancement of about $5 \%$ above unity (dashed line) of data (symbols) and simulations (solid line) for relative momenta $q<50 \mathrm{MeV} / \mathrm{c}$. For low values, $q<20 \mathrm{MeV} / \mathrm{c}$, this enhancement becomes suppressed by the Coulomb repulsion between the protons. Although the sensitivity of the directional cuts extends up to relative momenta of $q<50 \mathrm{MeV} / \mathrm{c}$, we restricted our fit region to lower values of $10 \leq q \leq 35 \mathrm{MeV} / \mathrm{c}$. In this region the shape of the simulated correlation functions describes well the data. The ratios in the right panel of Fig. 14 agree, however, similar well for the larger relative momenta. Using a fit region of $10 \leq q \leq 50 \mathrm{MeV} / \mathrm{c}$ yields radii within $\Delta R=0.2 \mathrm{fm}$ and emission times within $\Delta \tau=2 \mathrm{fm} / \mathrm{c}$ to those results extracted from the narrower relative momentum region.

\section{DISCUSSION}

The last collision of the two particles within the surrounding nuclear debris determines their freeze-out radius and is believed to occur when their mean free path gets larger than the system itself: the system becomes below a certain density transparent. We used reconstructed residue masses [13] to calculate the freeze out densities (Fig. 5). They are lower than the ground-state density of nuclei and therefore, show expansion. The strong variation of the freeze-out density is caused by the variation of the mass of the spectator. The freeze-out densities shown as open symbols were analysed in a previous work [5] with the assumption that the particles are emitted instantaneously. Correcting the source size with help of the directional cuts for the emission times of the protons results in densities (closed symbols) which are only slightly larger compared to the zero time assumption. The expected higher freeze-out densities were lowered by two effects, the collective movement of the source and by the initial Coulomb correlations within the source. In the left panel of Fig. 4 we show for $20 \leq Z_{B O U N D} \leq 40$ the simulation of a purely thermal source (thin lines) with the same radius. Guided by the freeze-out conditions of a statistical multifragmentation model [四] we tried to understand the variation of the freeze-out density with the impact parameter: assuming a constant crack width between the fragments, the freeze-out density becomes dependent on the multiplicity $M$. Hereby, the diameter of a

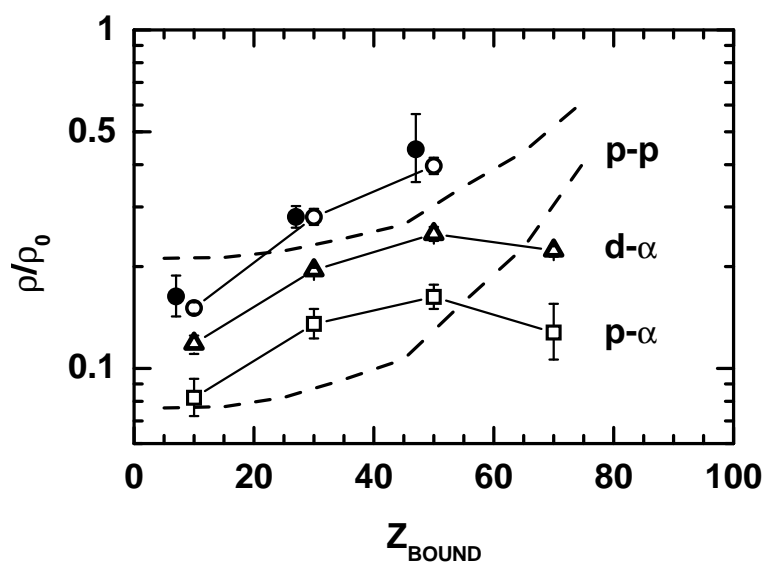

Figure 5. Freeze-out densities from p$\mathrm{p}, \mathrm{p}-\alpha$, and $\mathrm{d}-\alpha$ correlation functions (open symbols) were analysed assuming an instantaneous emission of the particles[5]. The closed symbols represent the freeze-out densities extracted from p-p correlation-functions assuming a Gaussian time distribution. The lines are explained in the text. 
nucleus in one dimension is compared to the diameter of an expanded nucleus with $M^{1 / 3}-1$ cracks with the width $2 d$. This one dimensional freeze-out radius is independent on the fragment size and only an approximation in three dimensions. In this simplified picture the freeze-out volume over the ground state volume is $V_{\text {freeze }} / V_{0}=\left(R_{0}+d\left(M^{1 / 3}-1\right)\right)^{3} / R_{0}^{3}$. We used the event multiplicity from Ref. [13] for different species of fragments. For the calculation of the primordial multiplicities we assume a simple sequential decay estimate: we observe in energy spectra for neutrons, protons, and alpha-particles a low energy component, which for the protons scales with the biggest charge in an event [15] and is likely due to sequential decays. We, therefore, select for those particles the high energy component of the spectra. For the crack width we assume two extreme assumptions [14]: for a gas of nucleons the crack width $2 d$ is in the order of $1.4 \mathrm{fm}$, the range of nuclear interaction. For an event with heavier fragments the fragments surfaces are separated by the distance where the nuclear attraction equals the Coulomb repulsion $(2 d=2.8 \mathrm{fm})$. Both assumptions are plotted in Fig. 5 as dashed lines. They exhibit the expected decrease of the freeze-out density with centrality. The extracted proton-proton freeze-out densities corrected for emission times follow closely within $20 \%$ the predictions for a crack width of $2 d=1.4 \mathrm{fm}$.

The other scattering states beside of the most peripheral reactions are in between of both predictions. These scattering states are, however, not corrected for emission times. The radius $R_{\tau=0}$ with inclusion of the emission times $\tau$ can be estimated from the uncorrected radius $R_{e f f}=\sqrt{R_{\tau=0}^{2}+\langle v \tau\rangle^{2}}$, with the thermal velocity $v$ for a fragment with temperature of $T=17 \mathrm{MeV}$ [15]. This estimate is plotted as solid and dashed lines in Fig. 3 for $Z_{B O U N D} \leq 20$ for radii from $\mathrm{p} \alpha$ - and $\mathrm{d} \alpha$-correlation functions, respectively. Emission times between $\tau=10-30 \mathrm{fm} / \mathrm{c}$ are needed to get a freeze-out radius comparable with that extracted from the emission-time corrected pp-analysis.

The times between the emission of protons are in the order of the passing time of the projectile through the target. We, therefore, cannot exclude that the protons come from the fireball, which grows with the centrality of the reaction. Attempts to fit the energy spectra in Fig. 2 with an fireball localized in momentum space failed. Nonetheless, the intriguing similarity of the impact-parameter dependence of the heavier scattering states $(\mathrm{p} \alpha, \mathrm{d} \alpha)$ which differ only by a common factor from the pp freeze-out densities indicate the emission from the target spectator.

It is interesting to point out that the average expansion from the RMS radius at ground-state to the freeze-out density within our emission times is much too high. It is $\langle\beta\rangle=0.19-0.29 \mathrm{c}$ for the most central bin and disagrees with the observed energy spectra. A low expansion velocity of about $\mathrm{E} / \mathrm{A}=0.5 \mathrm{MeV}(0.03 \mathrm{c})$, suggested by other studies [16], has the consequence that the nucleus first expands for $t \approx 100 \mathrm{fm} / \mathrm{c}$ and then decays within the extracted short times of $\tau=10-20 \mathrm{fm} / \mathrm{c}$. A reaction where the spectators are diluting each other by scattering out the fireball nucleons would explain the large radii without substantial amount of expansion. Calculating the expansion velocity of a nucleus starting to expand from the ground-state radius of a ${ }^{197} A u$ nucleus yields an average expansion velocity of $\langle\beta\rangle=0.04-0.09 \mathrm{c}$ for the most central bin. 


\section{CONCLUSION}

We constructed correlation functions for pairs of protons detected at backward angles in the reaction $\mathrm{Au}+\mathrm{Au}$ at $1000 \mathrm{AMeV}$ incident beam energy. Using high energy cuts of $E>20 \mathrm{MeV}$ we selected protons which are only little affected by sequential feeding [10]. Comparing the results with Monte-Carlo simulations within the Koonin-Pratt formalism fairly constant freeze-out radii of $R \approx 8 \mathrm{fm}$ are deduced and emission times of $\tau=10-15$ $\mathrm{fm} / \mathrm{c}$ are surprisingly short. The extracted radii are larger than the ground state radii of target spectators and show expansion. The mass dependence of the decaying target spectator with impact parameter lead to non constant freeze-out densities which decrease with centrality. Employing a multiplicity dependent freeze-out picture [14] we are able to describe the absolute value of the freeze-out densities as well as the impact parameter dependence. Because of the short emission times in the order of the passing time of both spectators we cannot exclude that the protons come from first stage scattering of the nuclear cascade. However the impact parameter dependence of the freeze-out densities of heavier scattering states from a former analysis [5, 10 indicates the emission from the target spectator. A more detailed analysis of these heavier scattering states is necessary to understand their formation and freeze-out properties.

\section{REFERENCES}

1. J. P. Bondorf, A. S. Botvina, A. S. Iljinov, I. N. Mishustin, and K. Sneppen. Phys. Rep., 257:133, 1995.

2. D. H. E. Gross. Phys. Rep., 279:119, 1997.

3. W. A. Friedman and W. G. Lynch. Phys. Rev. C, 28(1):16, 1983.

4. W. A. Friedman. Phys. Rev., C42:667, 1990.

5. S. Fritz et al., Phys. Lett. B, 461:315, 1999.

6. A. Schüttauf et al., Nuc. Phys, A607:457, 1996.

7. S. E. Koonin. Phys. Lett. B, 70(1):43, 1977.

8. S. Pratt and M.B. Tsang. Phys. Rev., C36:2390, 1987.

9. M. A. Lisa et al., . Phys. Rev. Lett, 71(18):2863, 1993.

10. C. Schwarz and S. Fritz. In H. Feldmeier, J. Knoll, W. Nörenberg, and J. Wambach, editors, Proceedings of the International Workshop XXVII on Gross Properties of Nuclei and Nuclear Excitations, page 168, Hirschegg, Austria, January, 17-23 1999. Gesellschaft für Schwerionenforschung mbH.

11. B. Kämpfer et al., Phys. Rev. C, 48(3):R955, 1993.

12. D. H. Boal. Ann. Rev. Nucl. Part. Sci., 37:541, 1987.

13. C. Groß. Anregungsenergien des Projektilrestkernes im System ${ }^{197}$ Au+ ${ }^{197}$ Au Kollisionen bei 600, 800 und 1000 AMeV. PhD thesis, J. W. Goethe Universität Frankfurt, 1997.

14. J. Bondorf, R. Donangelo, I. N. Mishustin, and H. Schulz. Nucl. Phys., A444:460, 1985.

15. T. Odeh et al., Phys. Rev. Lett, 84:4557, 2000.

16. L. Beaulieu et al., Phys. Rev. Lett, 84:5971, 2000. 